\title{
Strong correlation induced charge localization in antiferromagnets
}

SUBJECT AREAS:

SUPERCONDUCTING PROPERTIES AND

MATERIALS

THEORETICAL PHYSICS

Received

7 August 2013

Accepted

19 August 2013

Published

4 September 2013

Correspondence and requests for materials should be addressed to Z.-Y.W. (weng@ tsinghua.edu.cn)

\author{
Zheng Zhu', Hong-Chen Jiang' ${ }^{2}$, Yang Qi', Chushun Tian' \& Zheng-Yu Weng'
}

${ }^{1}$ Institute for Advanced Study, Tsinghua University, Beijing, 100084, China, ${ }^{2}$ Kavli Institute for Theoretical Physics, University of California, Santa Barbara, CA, $93106-4030$, U.S.A.

The fate of a hole injected in an antiferromagnet is an outstanding issue of strongly correlated physics. It provides important insights into doped Mott insulators closely related to high-temperature superconductivity. Here, we report a systematic numerical study of $t$ - $J$ ladder systems based on the density matrix renormalization group. It reveals a surprising result for the single hole's motion in an otherwise well-understood undoped system. Specifically, we find that the common belief of quasiparticle picture is invalidated by the self-localization of the doped hole. In contrast to Anderson localization caused by disorders, the charge localization discovered here is an entirely new phenomenon purely of strong correlation origin. It results from destructive quantum interference of novel signs picked up by the hole, and since the same effect is of a generic feature of doped Mott physics, our findings unveil a new paradigm which may go beyond the single hole doped system.

A critical step in understanding the physics of doped Mott insulators which is closely related to hightemperature superconductivity in cuprates ${ }^{1-3}$ is to investigate the motion of a single hole in Mott antiferromagnets $^{2-20}$. It is easy to imagine that an injected hole in an antiferromagnetically ordered state leaves on its path the trace of spin mismatches in the staggered magnetization direction (to be called " $S^{z}$-string" below). Such $S^{z}$-string was expected ${ }^{21}$ to cause the localization of the hole, but, as was later realized ${ }^{8}$, it can be self-healed via quantum spin flips. It was then conjectured ${ }^{7,8,10,11}$ that a quasiparticle picture should be still valid in longdistance, as if the Bloch theorem holds true even for such a many-body system. The quasiparticle picture was further supported by finite-size exact diagonalizations ${ }^{12}$ on lattices up to 32 sites $^{13}$. However, experimentally, in angle-resolved photoemission spectroscopy (ARPES) studies ${ }^{22-24}$, very broad single-particle spectral features have been observed in parent Mott insulator materials such as $\mathrm{Ca}_{2} \mathrm{CuO}_{2} \mathrm{Cl}_{2}$ and $\mathrm{Sr}_{2} \mathrm{CuO}_{2} \mathrm{Cl}_{2}$. They cast a serious doubt $^{14,17}$ on the rationale of understanding an individual hole created by the photon within a quasiparticle description. Recently, a strongly localized charge state in the copper-oxide layer, induced by an off-the-layer dopant defect, was observed in the scanning tunneling microscope experiment on $\mathrm{Ca}_{2} \mathrm{CuO}_{2} \mathrm{Cl}_{2}{ }^{25}$. In fact, the charge localization is generally found in a lightly doped cuprate before the system becomes a high- $T_{c}$ superconductor $^{26}$. These experiments raise a fundamentally important issue, i.e., whether the observed localization is an intrinsic phenomenon.

Theoretically, the validity of a quasiparticle description has been questioned in the literature as well. It was first argued by Anderson ${ }^{9}$ on general grounds that the quasiparticle spectral weight should vanish due to an unrenomalizable many-body phase shift induced when a hole is injected into a Mott insulator. Later, the discovery of the so-called phase string ${ }^{4,5,27}$ leads to a microscopic justification of this crucial observation. Here, a phase string may be regarded as an $S^{ \pm}$-string as opposed to the aforementioned " $S^{z}$-string”. It is irreparable by quantum spin flips in contrast to the $S^{z}$-string. It was further predicted ${ }^{17}$ that the phase string, instead of the $S^{z}$-string ${ }^{21}$, is responsible for an intrinsic self-localization of the injected hole in two dimensions.

In this work, a large-size numerical simulation is used as a powerful machinery to resolve this issue. This approach is of particular importance because the hole's motion is highly sensitive to quantum interference developing at long distance. To this end, we study the ladder systems by the DMRG method ${ }^{28}$, in which the length scale along one direction can be sufficiently large. As a ladder sample is long enough, we find that the charge generally gets localized so long as the leg number is larger than one. The localization scale decreases monotonically as the leg number increases. Contrary to this, if the sample is short, the injected hole behaves itinerantly, implying that the quasiparticle picture found in earlier numerical studies is likely a small-size effect. We demonstrate that the charge self-localization finds its origin in quantum destructive interference of the phase string, a unique property of the $t-J$ model $^{4,5,27}$. In particular, we show that the novel localization phenomenon 
disappears once the phase string is turned off artificially in the simulation, where a well-defined quasiparticle description is recovered in consistency with the common wisdom ${ }^{8}$ of spin polaron picture.

\section{Results}

Model Hamiltonians. A prototypical doped Mott insulator is described by the $t-J$ Hamiltonian, $H_{t-J}=H_{t}+H_{J}$, with

$$
\begin{aligned}
& H_{t}=-t \sum_{\langle i j\rangle \sigma}\left(c_{i \sigma}^{\dagger} c_{j \sigma}+\text { h.c. }\right), \\
& H_{J}=J \sum_{\langle i j\rangle}\left(\mathbf{S}_{i} \cdot \mathbf{S}_{j}-\frac{1}{4} n_{i} n_{j}\right) .
\end{aligned}
$$

Here, $c_{i \sigma}^{\dagger}$ is the electron creation operator at site $i, S_{i}$ the spin operator, and $n_{i}$ the number operator. The summation is over all the nearestneighbors, $\langle i j\rangle$. The Hilbert space is constrained by the no-doubleoccupancy condition, i.e., $n_{i} \leq 1$. At half-filling, $n_{i}=1$, the system reduces to Mott insulators (antiferromagnets) with a superexchange coupling, J. Upon doping a hole into this system, $\sum_{i} n_{i}=N-1(N$ the number of the lattice sites), and the hopping process is triggered as described by the hopping term, $H_{t}$, with $t$ the hopping integral.

For the single hole doped $t-J$ model in a bipartite lattice, there exists an exact theorem, which states ${ }^{4,5,27}$ that the propagation of the hole is a superposition of quantum amplitudes of all the paths, with each path carrying a unique sign sequence known as the phase string, i.e.,

$$
(+1) \times(-1) \times(-1) \times \cdots .
$$

Here, the sign \pm on the right-hand side keeps track of an $\uparrow$ or $\downarrow$-spin exchanged with the hole at each step of hopping as illustrated in Fig. 1 (A).

The quantum interference of phase strings from different paths is strong and play the fundamental role in dictating the long-distance behavior of the hole. In order to uniquely pin down the effect of phase strings, we also introduce the so-called $\sigma \cdot t$-J model with the hopping term $H_{t}$ in Eq. (1) replaced by

$$
H_{\sigma^{\cdot} t}=-t \sum_{\langle i j\rangle \sigma} \sigma\left(c_{i \sigma}^{\dagger} c_{j \sigma}+\text { h.c. }\right),
$$

(i.e., an extra sign $\sigma= \pm 1$ is added). It is easy to show, following Refs. $4,5,27$, that the phase string (2) disappears, whereas the positive weight for each path remains unchanged [as illustrated in Fig. 1 (B)]. In other words, one can artificially switch on and off the phase string effect between the $t-J$ and $\sigma \cdot t-J$ models to study its novel consequences.

Previously the DMRG algorithm has already been used to study the $t$ - $J$ model in ladder systems at low hole doping ${ }^{16,29}$. Below we shall focus on the one hole case on bipartite lattices of $N=N_{x} \times N_{y}$, where $N_{x}$ and $N_{y}$ are the site numbers in the $x$ and $y$ directions, respectively. By using the DMRG method, we shall study the ladders with small $N_{y}$ (from 1 to 5 ) and sufficiently large $N_{x}$. Here we set $J$ as the unit of energy and focus on the $t / J=3$ case unless otherwise specifically stated.

Self-localization of the charge. Typical examples of the hole density distribution, $\left\langle n_{i}^{h}\right\rangle \equiv 1-\left\langle n_{i}\right\rangle$, are shown in Fig. 2 (A) and (B) for $N_{y}=$ 3 and 4 , respectively. Here the charge profiles are plotted along the $x$ direction for a middle leg of the ladders. They are localized in the central region of the ladders with open boundaries. Upon summing up the distribution at all the sites of different legs, the sum rule: $\sum_{i}\left\langle n_{i}^{h}\right\rangle=1$ is satisfied. Examples of the contour plot of $\left\langle n_{i}^{h}\right\rangle$ in the $x-y$ plane can be found in Fig. S3 in Supplementary Information.

We systematically change the ladder length $N_{x}$ to study the full width at half-maximum (FWHM) of the charge profile. Fig. 2 (C)
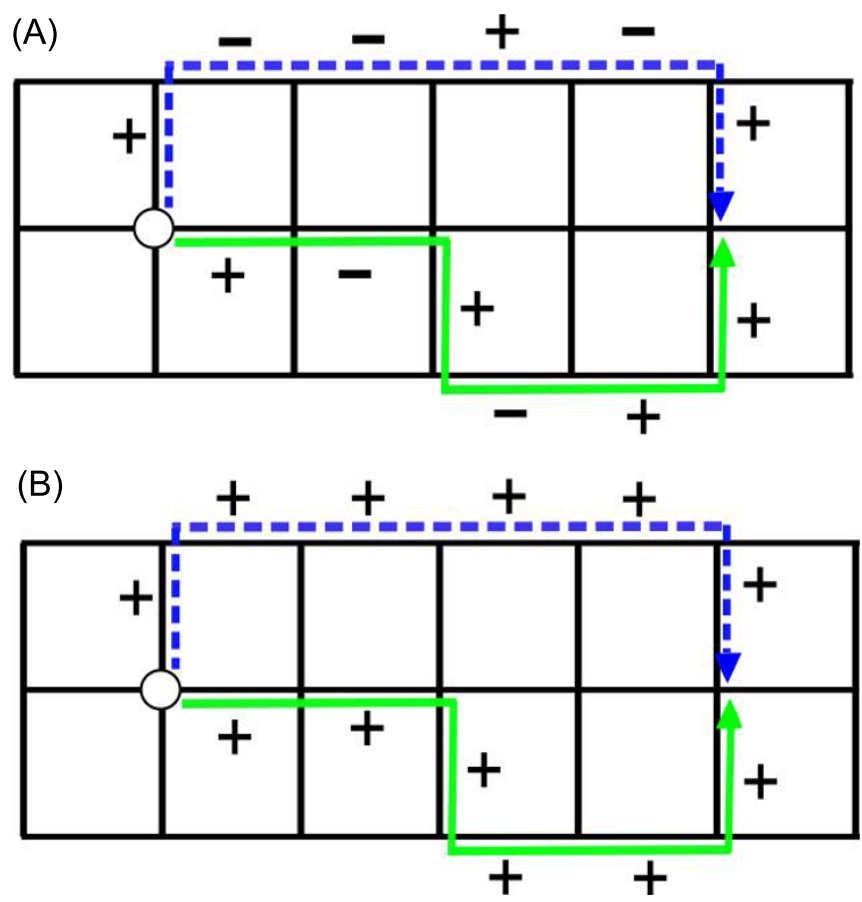

Figure $1 \mid$ (A) Quatum interference picture of one hole in the $t$-J model: As the hole moves from the injection point to a distant site, a sign sequence the phase string - is left behind as exemplified by the solid (green) and dashed (blue) lines. Here each $+(-)$ sign faithfully records an event that the hole exchanges its position with the nearest neighboring $\uparrow(\downarrow)$ spin during its motion. (B) By contrast, the phase string can be wholly switched off in the $\sigma \cdot t$-J model (see the text) such that the amplitude associated with each path is positive definite (of the same absolute magnitude as in the $t-J$ model). The presence (absence) of phase strings will drastically alter the long-wavelength behavior of the hole via quantum destructive (constructive) interference as demonstrated in this work.

shows that for ladders with different $N_{y}$, the FWHM increases linearly for small sample lengths, while saturates for sufficiently large sample lengths (except the $N_{y}=1$ case). The former indicates that the doped hole still remains itinerant at small $N_{x}$. The latter suggests that a self-localization takes place for the doped hole, when the ladder length is long enough where the FWHM is no longer sensitive to the boundaries along the $x$-direction. Later on, by a more precise analysis, it will be shown that such localization only involves the charge of the doped hole, not its spin. Furthermore, Fig. 2 (C) clearly shows that the saturated FWHM at $N_{y}>1$ monotonically decreases with the increase of the leg number $N_{y}$, implying that the localization effect becomes stronger and stronger. By contrast, there is no indication of saturation in FWHM for long one-dimensional chains $\left(N_{y}=\right.$ 1 , with $N_{x}$ up to 500), which is consistent with the fact that the doped hole in strictly one dimension exhibits the Luttinger liquid behavior ${ }^{2}$.

The destructive quantum interference effect of phase strings in the $t-J$ model provides a natural explanation for the charge "self-localization" observed in the above DMRG results. To further confirm the phase string origin of self-localization, we switch off the phase string interference by two methods and then repeat the previous procedure of numerical simulations. In the first method, we study the $\sigma \cdot t-J$ model introduced above. In the second method, we set the interchain hopping coefficient, $t_{\perp}$, to be zero such that the hole can only move in the $x$-direction as if in a one-dimensional chain. In both cases, the interference picture shown in Fig. 1 (A) breaks down. The numerical results for these two models are presented in Fig. 2 (D) for two-and three-leg cases, in which the FWHM of the hole density distribution restores the behavior of linear increase with the sample length. It 

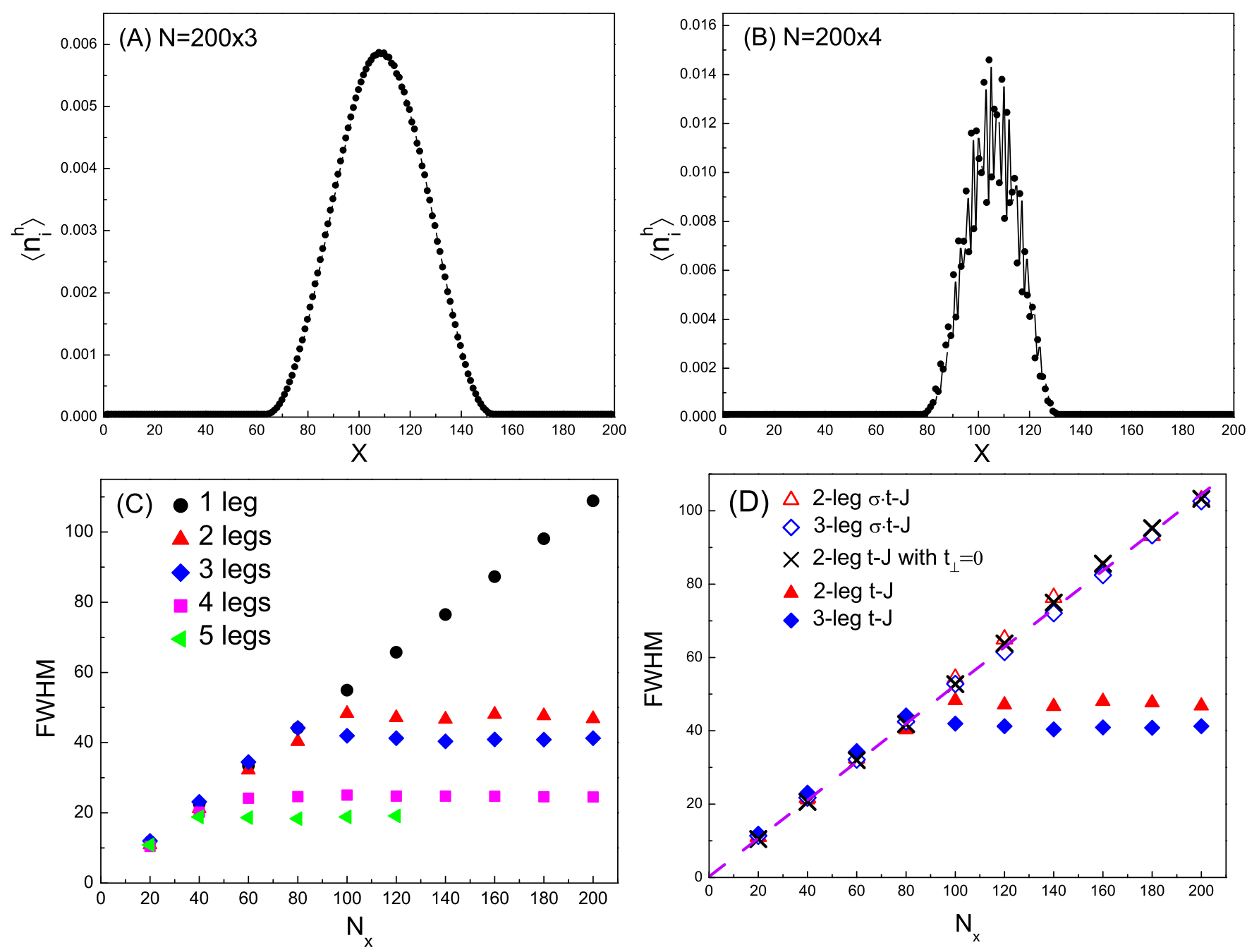

Figure $2 \mid$ Self-localization of the charge in a 3-leg ladder system of size $N=200 \times 3$ (A) and 4-leg ladder of $N=200 \times 4$ (B) with open boundaries. The density distribution $\left\langle n_{i}^{h}\right\rangle$ along the $x$ axis in a middle leg is well fitted by the Gaussian function with a full width at half-maximum (FWHM).

(C) The behavior of FWHM depends on the leg number $N_{y}$ : For the ladders of $N_{y}>1$, the FWHM first increases linearly at small $N_{x}$ and then saturates at large $N_{x}$, with a length scale monotonically decreasing with the leg number; for $N_{y}=1$, the FWHM grows unboundedly as the sample length increases. (D) Delocalization of the charge in the absence of phase string interference for the two modified models, namely the $\sigma \cdot t$-J model and the $t$-J model with interchain hopping coefficient $t_{\perp}=0$. The corresponding FWHM unboundedly increases with $N_{x}$ (open triangles, open diamonds, and crosses).

The dashed line $\left(=N_{x} / 2\right)$ is a guide to the eye.

indicates that the charge becomes delocalized in both cases, in sharp contrast to the self-localization in the $t$ - $J$ model.

Although the observed self-localization is insensitive to the parity (even-odd) of the leg number, the hole distribution is, as shown in Fig. 2 (A) and (B) and Supplementary Information: for the even-leg ladders $\left(N_{y}=2,4\right)$, there are always small spatial oscillations on top of the Gaussian density profile, while they are absent for the odd-leg ladders $\left(N_{y}=3,5\right)$. This can be attributed to distinct decaying behavior of spin-spin correlations for the odd- and even-leg ladders at half-filling: the former (latter) follows a power (an exponential) law reflecting the absence (presence) of spin gap, see Supplementary Information for further explanations. Furthermore, the distinction of the even-odd effect disappears with the increase of $t / J$ ratio (see Fig. S4), indicating that the detailed spin dynamics, governed by $J$, is not essential to the charge self-localization effect.

Momentum distribution. Now let us examine the momentum distribution of the hole by studying $n(\mathbf{k}) \equiv(1 / N) \sum_{i j} e^{i \mathbf{k} \cdot\left(\mathbf{r}_{i}-\mathbf{r}_{j}\right)} \sum_{\sigma}$ $\left\langle c_{i \sigma}^{\dagger} c_{j \sigma}\right\rangle$. At half-filling, one finds $n(\mathbf{k})=1$ and for the one hole case, $1-n(\mathbf{k})$ is shown in Fig. 3.
The insets of Fig. 3 (A) and (B) present the hole momentum distribution $1-n(\mathbf{k})$ as a function of $k_{x}$ for fixed $k_{y}=2 \pi / 3$ in the 3 -leg ladder and $k_{y}=\pi / 2$ in the 4-leg ladder, respectively. The value of $k_{y}$ is chosen in a way that the "sudden change" in $1-n(\mathbf{k})$ can reach maxima by varying $k_{x}$, according to the contour plots in the $k_{x}-k_{y}$ plane (see Supplementary Information). A very interesting feature is that after the rescaling: $1-n(\mathbf{k}) \rightarrow[1-n(\mathbf{k})] N$, all the curves in the inset of Fig. 3(A) [or (B)] collapse onto a universal curve shown in the corresponding main panel. If one defines the Fermi surface by the sudden jump in the momentum distribution function, then the two universal curves suggest that the quasiparticle spectral weight is upper-bounded by $1 / N$ for large $N$, and vanishes in the thermodynamic limit.

To satisfy the sum rule, $\sum_{\mathbf{k}}(1-n(\mathbf{k}))=1$, the width of the jump in $1-n(\mathbf{k})$ must remain finite in the limit $N \rightarrow \infty$. This is clearly shown in Fig. 3 (A) and (B), consistent with a finite localization length in the real space. We have also calculated the hole momentum distribution at different ratios of $t / J$. As shown in Supplementary Information, for a given sample size, the jump near the Fermi point is continuously reduced with increasing $t / J$, which is qualitatively consistent with earlier work ${ }^{12}$. 

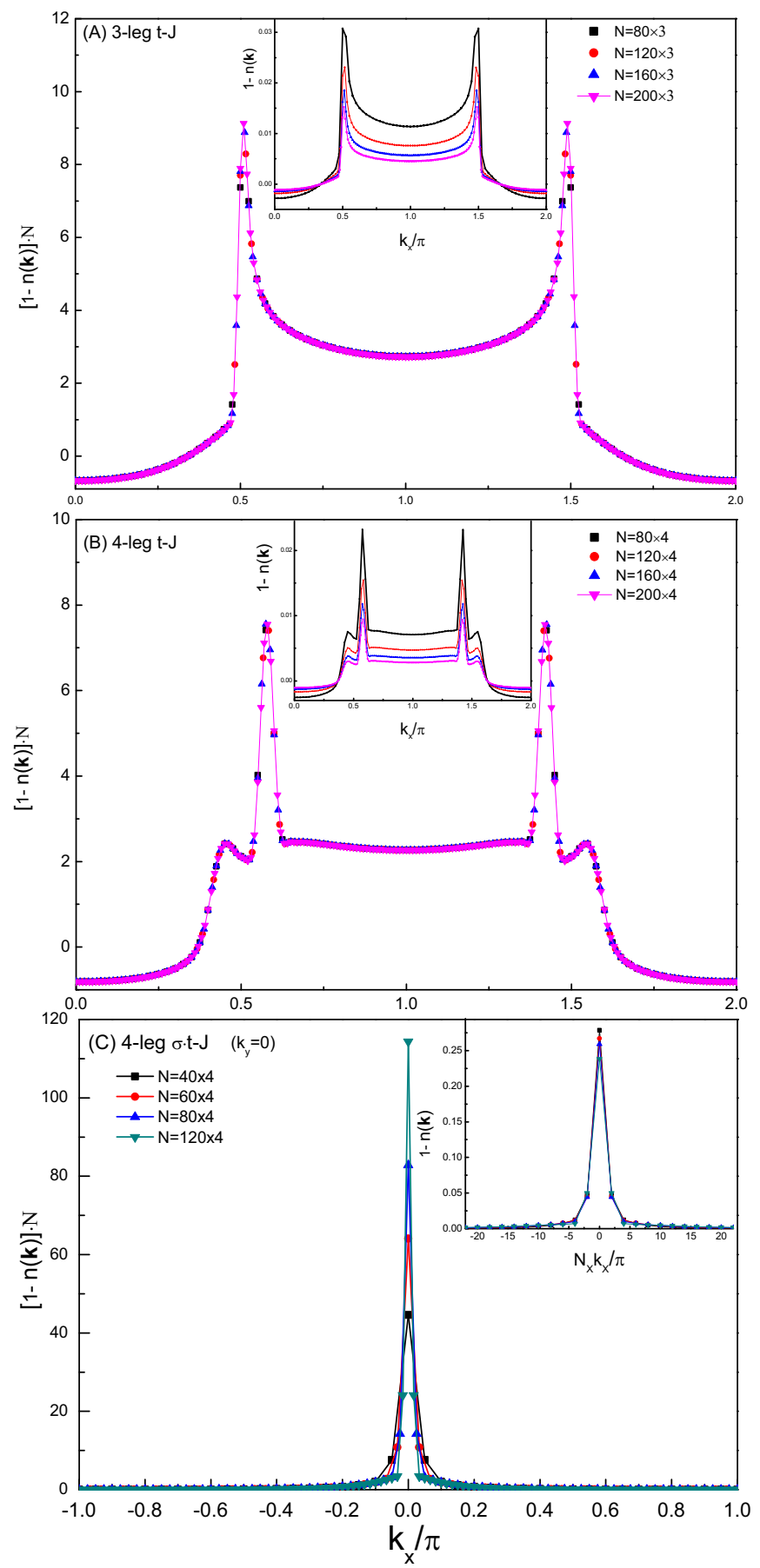

Figure 3 Momentum distribution. For both 3-leg (A) and 4-leg (B) ladders, the momentum distribution of the hole exhibits scaling behavior: After the rescaling, $1-n(\mathbf{k}) \rightarrow[1-n(\mathbf{k})] N$, the curves at different sample lengths in the inset collapse onto a single one in consistency with localization (main panels). Note that we fix $k_{y}=\frac{2 \pi}{3}$ for the 3 -leg case and $k_{y}=\frac{\pi}{2}$ for the 4-leg case, and plot the distribution along $k_{x}$. Representive contour plots in the whole $k_{x}-k_{y}$ plane are shown in Fig. S5 (Supplementary Information). (C) The quasiparticle picture is recovered for the $\sigma \cdot t-J$ model. The main panel shows that the momentum distributions of different sample lengths collapse to a single momentum at $k_{x}=k_{y}=0$ for the 4-leg ladder; The inset shows that a universal curve is obtained for $1-n(\mathbf{k})$ after the rescaling: $k_{x} \rightarrow N_{x} k_{x}$, which suggests a nonvanishing quasiparticle spectral weight peaked at $\mathbf{k}=0$.
Again a sharp contrast arises once the phase string is turned off in the $\sigma \cdot t$-J model, as shown in Fig. 3(C) for the 4-leg ladder. Here the wide spread of $1-n(\mathbf{k})$ in the momentum space seen in (A) and (B) collapses into a sharp peak at $k_{x}, k_{y}=0$. In particular, a universal curve (the inset) is obtained by the rescaling: $k_{x} \rightarrow N_{x} k_{x}$ instead of by $1-n(k) \rightarrow[1-n(\mathbf{k})] N$ as done in the main panel. This clearly indicates that the quasiparticle spectral weight is finite and a Blochlike state is restored with a definite momentum at $\mathbf{k}=0$ (a peak at the equivalent of $k_{x}, k_{y}=\pi$ is also found).

Spin degree of freedom. Complementary to the charge localization illustrated in Fig. 2, we further explore the fate of the spin-1/2 associated with the hole injected into the half-filled spin-singlet background.

For the 3-leg ladder, the spin-1/2 is explicitly separated from the charge as demonstrated by Fig. 4(A). Here the single hole is created by removing a $\downarrow$ spin electron. While the charge of the doped hole is well localized at the sample center, the extra spin of $S^{z}=1 / 2$ spreads over the sample. It is indicated by spin density $\left\langle S_{i}^{z}\right\rangle$ as well as the coarse grained $\left\langle S_{i}^{z}\right\rangle_{\text {c.g. }}$, which is approximately uniform across the ladder. This is probably the most direct display of electron fractionalization for a doped Mott insulator, thanks to the charge localization.

By contrast, for an even-leg ladder, because of the presence of a spin gap in the charge-free spin background, the extra spin of $S^{z}=1 /$ 2 tends to stay in the hole region in order to reduce its superexchange energy cost, as illustrated in Fig. 4(B) for the 2-leg ladder case. In order to examine properly how the charge and spin associated with the doped hole behave, one has to resort to a different method.

Note that, first, if the charge part - namely the holon - is localized, the localization center can be anywhere in a translationally invariant system. In particular, an "extended" profile as a linear superposition of the localized wave packets at different positions should be also energetically degenerate. Second, if Fig. 4(B) really corresponds to spin-charge separation, the extra spin-1/2 - namely the spinon should be more or less "free" within the regime expanded by the charge distribution. Then the spinon will prefer a more extended distribution of the hole to further lower its energy. In other words, the degeneracy of localized states should be lifted in favor of an extended profile in order to gain additional spinon energy.

Indeed, by increasing DMRG sweep number with the truncation error reaching much lower than $10^{-8}$, the localized profile in Fig. 4(B) is found to slowly converge to an extended one as shown in (C) with the total energy lowered by $\sim 10^{-4} \mathrm{~J}$. To identify the nature of this small energy gain, one may vary the sample size from $N_{x}=16$ to 800 , and repeat the same procedure to obtain the one-hole energy $E_{G}^{1-\text { hole }}$ at each $N_{x}$ [with the same full convergence to the extended charge profile as in Fig. 4(C)]. As shown in the inset of Fig. 5(A), with a constant term excluded, $E_{G}^{1-\text { hole }}$ is well fitted by $\alpha\left(\pi / N_{x}\right)^{2}$ (blue triangles) with the coefficient $\alpha=0.87 \mathrm{~J}$, as if it is contributed by some "free particle" in a box of length $N_{x}{ }^{30}$.

Next, we show that such $1 / N_{x}^{2}$ (finite-size) contribution to $E_{G}^{1-\text { hole }}$ is not from the charge sector. For this purpose, we make the 2-leg ladder as a ribbon by connecting the boundaries on the two edges of the ladder in the $x$-direction. Then we calculate the energy difference $\Delta E_{G}^{1-\text { hole }} \equiv E_{G}^{1 \text {-hole }}(\pi)-E_{G}^{1-\text { hole }}(0)$ with threading through a flux $\pi$ into the ribbon. It corresponds to the change of boundary condition from the periodic to anti-periodic one for the hopping term and is equivalent to coupling a small electric field to the charge sector. As shown in Fig. 5(A), $\Delta E_{G}^{1-\text { hole }}$ oscillates strongly and falls off exponentially as $e^{-N_{x} / \xi}$ with $\xi \sim 14.5$. It behaves completely different from $1 / N_{x}^{2}$ behavior exhibited in $E_{G}^{1-h o l e}$, indicating that the charge indeed remains localized. The "free particle-like" behavior in $E_{G}^{1-h o l e}$ should therefore solely originate from the charge-neutral part of the doped hole, i.e., the spinon. 

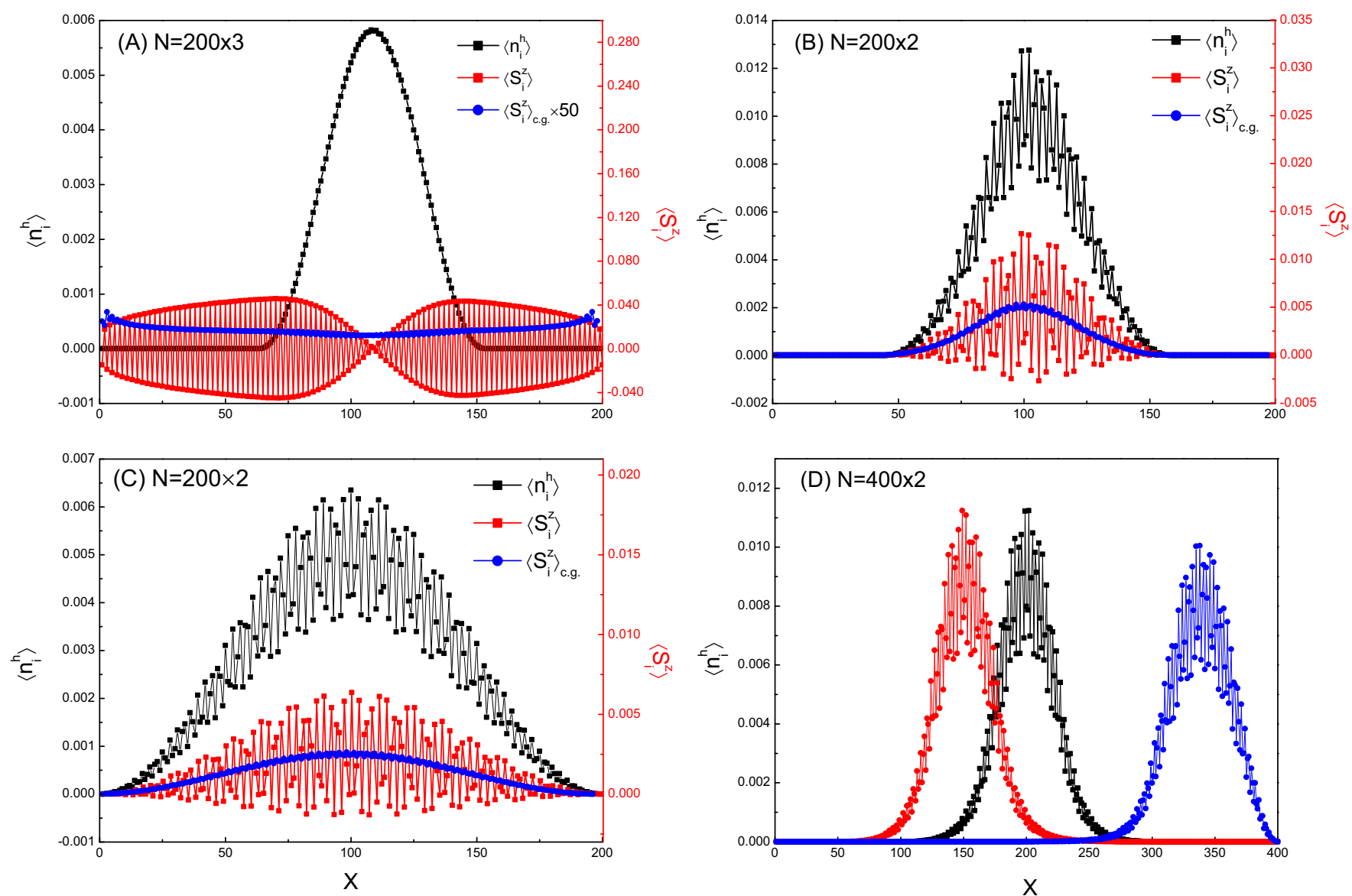

Figure $4 \mid$ (A) Spin-charge separation in a 3-leg ladder with a $\downarrow$ spin electron removed: The charge of the hole is localized at the sample center and the spin of $S^{z}=1 / 2$ spreads over the entire sample, with the coarse grained density $\left\langle S_{i}^{z}\right\rangle c$.g. approximately uniform. (B) In a 2-leg ladder, the charge and spin of the doped hole remain in the same localized region because of a finite spin-gap in the spin background. (C) An extended profile of the charge can be obtained with further reducing DMRG truncation error (see the text); In this case, although the charge remains localized, the mobile spinon gains a tiny additional energy (cf. Fig. 5). (D) In a $N=400 \times 2$ ladder, by adding weak chemical potentials the hole density can be localized anywhere along the ladder.

By sharp contrast, upon turning off the phase-string in the $\sigma \cdot t-J$ model, both $E_{G}^{1-\text {-hole }}$ and $\Delta E_{G}^{1-\text { hole }}$ follow the same $1 / N_{x}^{2}$ behavior as clearly shown in the inset of Fig. 5(A). It is consistent with the previous conclusion that in this case the doped hole is restored as a quasiparticle, which carries both charge and spin and thus responds very similarly to the boundary condition (i.e., the finite-size effect) as a single mobile object.

The degenerate charge localized states can be superposed to form a more "extended" distribution to minimize the spinon energy due to the residual spin-charge interaction,. It has been shown ${ }^{31}$ that generally the DMRG method tends to pick up a minimally entangled state among quasi-degenerate states. Thus, spatially localized ones such as those in Fig. 2 are naturally obtained because a further spinon energy gain for a bigger FWHM would be too tiny to be detected with a given small DMRG truncation error. It also explains why the FWHM of charge distribution shown in Figs. 2 and 4 is usually bigger than the intrinsic localization length $\xi$ as exhibited by $\Delta E_{G}^{1 \text {-hole }}$. But the former is still valid to characterize the general trend of the localization, say, as a function of the leg number in Fig. 2. On the other hand, by adding some weak local chemical potentials, the quasidegeneracy of the hole states can be lifted such that the hole density profile is truly localized around the impurities at different locations [as indicated by different colored curves in Fig. 4(D) for a $N=400 \times$ 2 ladder]. Here the increase of the total energy of the original $t-J$ Hamiltonian is weak (about $10^{-4} \mathrm{~J}$ ), presumably from the spinon part as the FWHM is still larger than $\xi$.
Finally, the spin-charge separation is well-known for the onedimensional chain ${ }^{2}$. Figure 5(B) presents the corresponding 1-leg results. As shown in the inset, the finite-size effect of $E_{G}^{1-\text {-hole }}\left(\propto 1 / N_{x}^{1.5}\right)$ is indistinguishable from the phase string free case (with open boundary conditions). But the charge response of $E_{G}^{1-\text { hole }}$ is again totally different [the main panel of Fig. 5(B)]. It oscillates strongly as a function of $N_{x}$, similar to the 2-leg case in Fig. 5(A). As a matter of fact, it can be directly attributed to the phase string modulation in the expression for the ground state energy given in Ref. 17. The main difference from the 2-leg case is that the envelop of $\Delta E_{G}^{1-\text { hole }}$ decays with increasing $N_{x}$ in a power law fashion $\left(\propto 1 / N_{x}^{3}\right)$ instead of an exponential one. This is consistent with the fact that the holon is not localized in the one-dimensional case. It is interesting to notice that even without the phase string effect, $E_{G}^{1 \text {-hole }}$ and $\Delta E_{G}^{1 \text {-hole }}$ behave distinctly for the $\sigma \cdot t-J$ model. The former $\propto 1 / N_{x}^{1.5}$, while the latter $\propto 1 / N_{x}^{2}$, also indicating a spin-charge separation although the distinction is much less dramatic as compared to the $t-J$ model.

\section{Discussion}

Charge self-localization is a striking result of a single-hole-doped Mott insulator. However, for this novel effect to get fully unveiled, a large sample is needed. Instead of tackling directly a two-dimensional square lattice, in this work, we have chosen ladders that the DMRG method can access. As it turns out, very surprising results show up so long as the ladders are long enough, and, there are more 

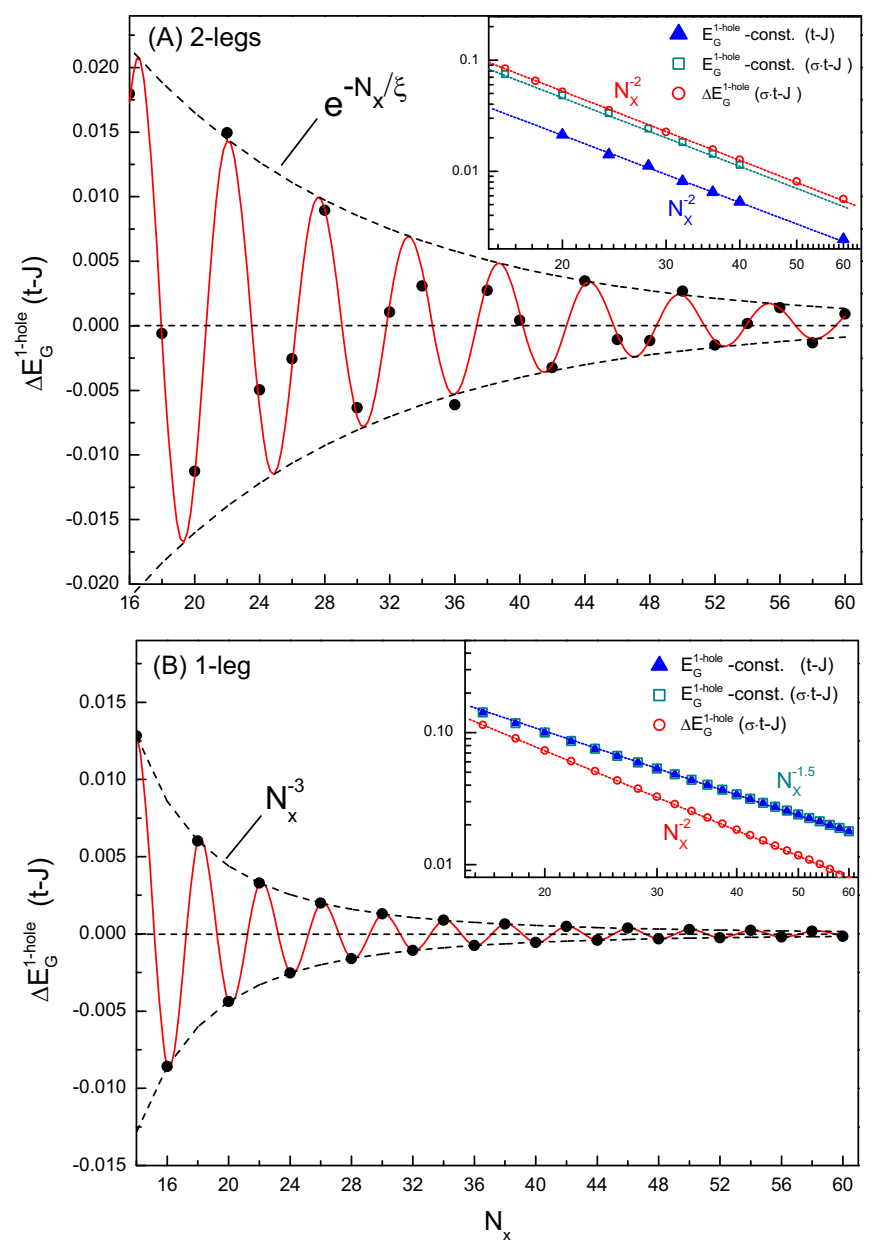

Figure $5 \mid$ The energy difference $\Delta E_{G}^{1-h o l e}$ between anti-periodic and periodic boundary conditions along the $x$-direction for the charge shows an oscillation in $N_{x}$, which is a direct manifestation of the phase string effect. The exponential decay of the envelop (dashed curves) of $\Delta E_{G}^{1-\text { hole }}$ shows the charge localization for the 2-leg ladder (A), while the power law decay for the 1-leg ladder case (B) indicates a Luttinger liquid behavior. By sharp contrast, in the inset of (A), a completely different finite-size behavior $\left(1 / N_{x}^{2}\right)$ is exhibited in the ground state energy $E_{G}^{1-\text { hole }}$ for the 2leg ladder, confirming the spin-charge separation (see the text). For comparison, both $\Delta E_{G}^{1-\text { hole }}$ and $E_{G}^{1-\text { hole }}$ follow the same $1 / N_{x}^{2}$-dependence once the phase string is turned off, as shown in the inset of $(\mathrm{A})$, where the quasiparticle picture is restored with a spin-charge recombination. In the inset of (B), similar comparisons for the 1-leg case are presented.

than one paths to realize quantum interference, e.g., in a two-leg system.

The most essential physics underlying the observed exotic phenomena is that the motion of the single hole acquires a sequence of signs that precisely and permanently record each microscopic holehopping-spin-backflow event in the quantum spin background. These irreparable phase strings cause singular and destructive interference of quantum waves of the charge. As the distance of wave propagation increases, effects of destructive interference become stronger and stronger. The present results show unequivocally that the self-localization of the charge, irrespective of even or odd ladders, is a combined effect of quantum interference and multiple scattering between the doped charge and spin background, in analogy to Anderson localization $^{32}$. This analogy notwithstanding, their nature is fundamentally different: The charge self-localization arises purely from strong electron correlation in clean systems; whereas Anderson localization from disorders in noninteracting electron systems.
Consistent with the picture of charge self-localization above, in the single chain case, since quantum interference involving different paths is absent, the doped charge is delocalized.

The irreparable phase string effect is fundamentally different from the premise for a spin polaron picture - a conventional wisdom in which the disturbance of the hole hopping to the spin background is described by virtual spin excitations that eventually relax back after the hole moves away. In fact, the spin-polaron or quasiparticle behavior has been shown to be recovered once the phase string is "switched off" in the so-called $\sigma \cdot t$-J model, where everything seems "normal" in a conventional sense. Here, a spin-charge recombination with a good kinetic energy for the quasiparticle can be realized since there is no more singular phase string effect to strongly suppress the kinetic energy of the charge.

Phase strings have been proved ${ }^{27}$ rigorously as the sole sign structure in the $t-J$ model on bipartite lattices, regardless of doping concentration, temperature, and dimensions. As such, the present work may provide significant insights into the long-standing issue of high temperature superconductivity in the cuprates at finite doping. One may point out that for the realistic materials, the $t-J$ model needs modified. For example, a non-bipartite lattice with a finite nextnearest-neighboring hopping integral $t^{\prime}$ or the Hubbard model with an intermediate on-site repulsion $U$ is probably more relevant than the simpliest $t$ - $J$ model. We have numerically checked that the singlehole localization remains robust so long as $t^{\prime}$ is small (e.g., $t^{\prime} / t<0.2$ for the 3-leg ladder at $t=3 \mathrm{~J}$ ), or in a Hubbard model, at least, with $U / t>10$. It suggests that the generic localization found ${ }^{26}$ in the lightly doped cuprates may intrinsically belong to the new paradigm revealed in this work.

\section{Methods}

The numerical simulations in this work are performed by using the standard DMRG method. For Fig. 5, because the systems involved are small, we performed the simulation for both the open and periodic boundary conditions. For other results, the systems involved are large and we adopted the open boundary condition due to the numerical difficulty. In DMRG simulations, we keep up to $m=5000$ states in the DMRG block with up to 40 sweeps to get converged results. For the measurement of the ground-state energy and other observables, the total truncation error is of the order or less than $10^{-8} \sim 10^{-12}$.

To examine the validity of our numerical method, we calculated the ground-state energy for small systems and compared it with that obtained by the exact diagonalization method. The results are in excellent agreement. We also calculated the spinspin correlation functions of the Heisenberg ladders (half-filled $t$ - $J$ ladders). The results are consistent with those reported in earlier works (see Supplementary Information for details).

1. Anderson, P. W. The resonating valence bond state in $\mathrm{La}_{2} \mathrm{CuO}_{4}$ and superconductivity. Science 235, 1196 (1987).

2. Anderson, P. W. The Theory of Superconductivity in the High-Tc Cuprates (Princeton University Press, Princeton, NJ, 1997).

3. Lee, P. A., Nagaosa, N. \& Wen, X. G. Doping a Mott insulator: Physics of hightemperature superconductivity. Rev. Mod. Phys. 78, 17 (2006).

4. Sheng, D. N., Chen, Y. C. \& Weng, Z. Y. Phase string effect in a doped antiferromagnet. Phys. Rev. Lett. 77, 5102 (1996).

5. Weng, Z. Y., Sheng, D. N., Chen, Y. C. \& Ting, C. S. Phase string effect in the t-J model: General theory. Phys. Rev. B 55, 3894 (1997).

6. Shraiman, B. I. \& Siggia, E. D. Mobile Vacancies in a Quantum Heisenberg Antiferromagnet. Phys. Rev. Lett. 61, 467 (1988).

7. Schmitt-Rink, S., Varma, C. M. \& Ruckenstein, A. E. Spectral function of holes in a quantum Antiferromagnet. Phys. Rev. Lett. 60, 2793 (1988).

8. Kane, C. L., Lee, P. A. \& Read, N. Motion of a single hole in a quantum antiferromagnet. Phys. Rev. B 39, 6880 (1989).

9. Anderson, P. W. "Luttinger-liquid" behavior of the normal metallic state of the $2 \mathrm{D}$ Hubbard model. Phys. Rev. Lett. 64, 1839 (1990).

10. Martinez, G. \& Horsch, P. Spin polarons in the t-J model. Phys. Rev. B 44, 317 (1991).

11. Liu, Z. \& Manousakis, E. Spectral function of a hole in the t-J model. Phys. Rev. B 44, 2414 (1991).

12. Dagotto, E. Correlated electrons in high-temperature superconductors. Rev. Mod. Phys. 66, 763 (1994).

13. Leung, P. W. \& Gooding, R. J. Dynamical properties of the single-hole t-J model on a 32-site square lattice. Phys. Rev. B 52, R15711 (1995).

14. Laughlin, R. B. Evidence for quasiparticle decay in photoemission from underdoped cuprates. Phys. Rev. Lett. 79, 1726 (1997). 
15. Lee, T. K. \& Shih, C. T. Dispersion of a single hole in the t-J model. Phys. Rev. B 55 , 5983 (1997).

16. White, S. R. \& Scalapino, D. J. Hole and pair structures in the t-J model. Phys. Rev. $B$ 55, 6504 (1997).

17. Weng, Z. Y., Muthukumar, V. N., Sheng, D. N. \& Ting, C. S. Spin-charge separation in the single-hole-doped Mott antiferromagnet. Phys. Rev. B 63 075102 (2001).

18. Mishchenko, A. S. \& Nagaosa, N. Electron-Phonon Coupling and a Polaron in the t-J Model: From the Weak to the Strong Coupling Regime. Phys. Rev. Lett. 93, $036402(2004)$

19. Brunner, M., Assaad, F. F. \& Muramatsu, A. Single-hole dynamics in the t-J model on a square lattice. Phys. Rev. B 62, 15480 (2000).

20. Mishchenko, A. S., Prokof'ev, N. V. \& Svistunov, B. V. Single-hole spectral function and spin-charge separation in the t-J model. Phys. Rev. B 64, 033101 (2001).

21. Bulaevskii, L. N., Nagaev, E. L. \& Khomskii, D. L. A new type of auto-localized state of a conduction electron. Sov. Phys. JETP 27, 836 (1968).

22. Wells, B. O., Shen, Z. X., Matsuura, A., King, D. M., Kastner, M. A., Greven, M. \& Birgeneau, R. J. E versus k Relations and Many Body Effects in the Model Insulating Copper Oxide $\mathrm{Sr}_{2} \mathrm{CuO}_{2} \mathrm{Cl}_{2}$. Phys. Rev. Lett. 74, 964 (1995).

23. Ronning, F., Kim, C., Feng, D. L., Marshall, D. S., Loeser, A. G., Miller, L. L., Eckstein, J. N., Bozovic, I. \& Shen, Z. X. Photoemission evidence for a remnant Fermi surface and a d-wave-like dispersion in insulating $\mathrm{Ca}_{2} \mathrm{CuO}_{2} \mathrm{Cl}_{2}$. Science 282, 2067 (1998).

24. Shen, K. M., Ronning, F., Lu, D. H., Lee, W. S., Ingle, N. J. C., Meevasana, W., Baumberger, F., Damascelli, A., Armitage, N. P., Miller, L. L., Kohsaka, Y., Azuma, M., Takano, M., Takagi, H. \& Shen, Z.-X. Missing quasiparticles and the chemical potential puzzle in the doping evolution of the cuprate superconductors. Phys. Rev. Lett. 93, 267002 (2004).

25. Ye, C., Cai, P., Yu, R. Z., Zhou, X. D., Ruan, W., Liu, Q. Q., Jin, C. Q. \& Wang, Y. Y. Visualizing the atomic scale electronic structure of the $\mathrm{Ca}_{2} \mathrm{CuO}_{2} \mathrm{Cl}_{2}$ Mott insulator. Nature Communications 4, 1365 (2013).

26. Ando, Y., Lavrov, A. N., Komiya, S., Segawa, K. \& Sun, X. F. Mobility of the doped holes and the antiferromagnetic correlations in underdoped high-Tc cuprates. Phys. Rev. Lett. 87, 017001 (2001).

27. Wu, K., Weng, Z. Y. \& Zaanen, J. Sign structure of the t-J model. Phys. Rev. B 77, $155102(2008)$

28. White, S. R. Density matrix formulation for quantum renormalization groups. Phys. Rev. Lett. 69, 2863 (1992)
29. White, S. R. \& Scalapino, D. J. Ground states of the doped four-leg t-J ladder. Phys. Rev. B 55, R14701 (1997).

30. White, S. R. priviate communication.

31. Jiang, H. C., Wang, Z. \& Balents, L. Identifying topological order by entanglement entropy. Nature Physics 8, 902-905 (2012).

32. Anderson, P. W. Absence of diffusion in certain random lattices. Phys. Rev. 109, 1492 (1958).

\section{Acknowledgements}

Stimulating discussions with L. Balents, D. N. Sheng, S. R. White, J. Zaanen, and especially P. A. Lee are acknowledged. This work was partially supported by the KITP (NSF no. PHY05-51164) and the Center for Scientific Computing at the CNSI and MRL: the NSF MRSEC (DMR 1121053) and NSF CNS-0960316; by the NBRPC (nos. 2009CB929402, 2010CB923003, and 2011CBA00108); by the NSFC (no. 11174174) and the Tsinghua University ISRP (no. 2011Z02151).

\section{Author contributions}

H.C.J. developed the simulation codes, Z.Z. conducted the numerical experiments, Y.Q., C.T. and Z.Y.W. designed the project. All authors equally participated in discussing the results and writing the manuscript.

\section{Additional information}

Supplementary information accompanies this paper at http://www.nature.com/ scientificreports

Competing financial interests: The authors declare no competing financial interests.

How to cite this article: Zhu, Z., Jiang, H.-C., Qi, Y., Tian, C.S. \& Weng, Z.-Y. Strong correlation induced charge localization in antiferromagnets. Sci. Rep. 3, 2586; DOI:10.1038/ srep02586 (2013)

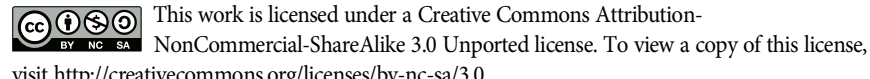
visit http://creativecommons.org/licenses/by-nc-sa/3.0 